\title{
SISTEMA DE AUXílio À OPERAÇÃo DE PEQUENAS CENTRAIS HIDRELÉTRICAS UTILIZANDO LÓGICA NEBULOSA
}

\author{
RONNIER F. ROHRICH ${ }^{1}$, ALESSANDRO ZIMMER ${ }^{2}$.
}

1. Departamento de Engenharia Elétrica, Universidade Federal do Paraná

UFPR - Setor de Tecnologia, Programa de Pós-Graduação em Engenharia Elétrica, Caixa Postal 19011 / Centro Politécnico, CEP 81531-970 / Curitiba PR Brasil

E-mails: ronnier.fratescyahoo.com.br

\author{
2. Departamento de Engenharia Elétrica, Universidade Federal do Paraná \\ UFPR - Setor de Tecnologia, Programa de Pós-Graduação em Engenharia Elétrica, Caixa Postal \\ 19011 / Centro Politécnico, CEP 81531-970 / Curitiba PR Brasil \\ E-mails: zimmerdeletrica.ufpr.br
}

\begin{abstract}
The main objective of this article is to present a methodology that helps the operation of Small Hydropower Plants (SHPP). A fuzzy system that suggests the outpot power setting based on the behavior of seven input variables was conceived for this purpose. The selection of the input variables as well as the evaluation of the system's performance was done through the use of real operational data obtained from a Small Hydropwer Plant during a one year time span. During the simulations, a significant increase on the power production was obtained when compared to the actual power plant operation.
\end{abstract}

Keywords— Small Hydropower Plant, Fuzzy Logic, Operation, Power Selected, Correlation.

Resumo- Este artigo tem como principal objetivo, apresentar um sistema de apoio à decisão que auxilie a operação de Pequenas Centrais Hidrelétricas (PCHs). Para tal objetivo, foi desenvolvido um sistema nebuloso que sugere uma regulagem da potência de saída baseado no comportamento de um conjunto de sete variáveis de entrada. A seleção destas variáveis, bem como a avaliação de desempenho do sistema foi feita através da utilização de dados reais da operação de uma Pequena Central Hidrelétrica coletados no período de um ano. Obteve-se nas simulações de operação um aumento percentual expressivo na produção de energia quando comparada com a operação real da mesma planta.

Palavras-chave— Pequena Central Hidrelétrica, Lógica Nebulosa, Operação, Potência Selecionada, Correlação.

\section{Introdução}

Pequenas Centrais Hidroelétricas, conhecidas como PCHs, são empreendimentos hidráulicos importantes para garantir a continuidade do fornecimento de energia elétrica no Brasil e a sua expansão. Devido às suas características, tais como, pequeno período de construção, alagamento de áreas reduzidas para a formação do reservatório, costumam receber incentivos do governo, sofrendo ao mesmo tempo uma menor resistência por parte dos órgãos ambientais que analisam os impactos causados à sua implantação.

Apesar de na última década terem ocorrido inúmeros avanços nessa área, a regulamentação desses empreendimentos não determina uma metodologia específica que a operação das PCHs devam seguir. Os procedimentos de rede do Operador Nacional do Sistema (ONS) são documentos que visam apenas o planejamento da operação eletroenergética do sistema interligado nacional, desde que não ocorra ônus ao ONS e a energia assegurada seja gerada conforme a Resolução Normativa $n^{\circ}$ 266, Aneel (2007), a operação fica a critério do empreendedor. Facultando assim que a mesma seja realizada de maneira displicente.

Existem inúmeras variáveis que norteiam a operação de uma Pequena Central Hidrelétrica, associada ao conhecimento de quem as opera. Dentre elas podem-se citar o nível do reservatório, vazão afluente, vazão efluente, vazão turbinada pelas Unidades $\mathrm{Ge}$ radoras, curva de colina das turbinas, nível do canal de fuga e pluviometria. A partir desses dados, são definidos os patamares de geração que cada unidade de uma PCH deverá atingir no decorrer de um dia. Depreende-se então que a gestão da operação de Pequenas Centrais feitas de uma maneira eficiente deve trazer ganhos reais na produção final de energia. Para tal foi desenvolvido um sistema de suporte à decisão que, baseado nos dados de operação disponíveis, ajusta a curva de potência desejada, informando o operador qual configuração deve aplicar a fim de melhorar o desempenho da planta.

Variáveis linguísticas - variáveis cujos valores são sentenças em uma linguagem normal ou artificial - Zadeh, (1973) são utilizadas diariamente pelas pessoas para classificar, estimar, analisar e definir diversas situações do cotidiano. A utilização desse concei- 
to facilita o entendimento e aplicação da modelagem, quando da necessidade em definir graus de pertinência diferentes para um conjunto de variáveis. Baseando-se nesses conceitos é possível obter uma abordagem aproximada e descrever os comportamentos de diversos sistemas complexos de serem modelados com uma matemática precisa.

\section{Metodologia}

Devido às características peculiares à operação de PCHs e a modelagem necessária que esse trabalho exigiu, foi optado pela utilização da Lógica Nebulosa, tendo em vista que Sistemas de Inteligência Artificial vem sendo empregados há muitos anos nas mais diversas áreas tecnológicas, e possuem métodos já sacramentados no meio acadêmico e na indústria.

Tem-se como objetivo principal deste trabalho, verificar os critérios utilizados por um operador de PCHs para tomar a decisão de seleção de potência fornecida pela unidade geradora durante certo intervalo de tempo. Isto feito, codifica-se este conhecimento em um sistema nebuloso esperando obter uma geração mais eficiente das unidades geradoras, levando-se em conta restrições operacionais e intervalos limitados para as variáveis de entrada e saída do sistema, como por exemplo, o nível mínimo que o reservatório pode atingir devido a eventuais restrições hídricas, os valores que compõe o diagrama de colina das unidades, isto é, a queda líquida, a vazão, a potência das turbinas, garantindo assim resultados que evitam uma degradação precoce das unidades geradoras.

O sistema desenvolvido neste trabalho, utiliza-se de uma metodologia composta de cinco etapas, sendo elas:

- Aquisição de dados;

- Definição do ambiente em que as variáveis linguísticas estão associadas ao sistema;

- Definição do número de termos primários e graus de pertinência dos conjuntos que envolvem cada variável;

- Formulação das regras que constituem o sistema nebuloso;

- Definição dos patamares em que o sistema será desenvolvido, o método de inferência e a forma de "defuzzificação" das variáveis incluindo a sua atuação no sistema nebuloso.

- Testes.

\subsection{Base de Dados}

Os dados utilizados neste trabalham foram coletados de uma Pequena Central Hidrelétrica as margens do Rio Chapecó que possui a capacidade de gerar até $30 \mathrm{MW}$ de energia elétrica, situada no estado de Santa Catarina. Os dados utilizados são referentes a operação da planta no período de 01/03/2012 a 28/02/2013, abrangendo todas particularidades que cada época do ano apresenta, como períodos de alta pluviometria e os períodos de estiagem na bacia do Rio Uruguai (bacia que o Rio Chapecó está situado). Os dados utilizados para elaboração das análises, simulações e comparações foram incluídos em um único banco de dados para que fosse facilitada a análise detalhada. Abaixo seguem as variáveis coletadas:

- Potência Selecionada pelo operador (MW);

- Vazão Afluente (m³/s);

- Variação no nível do reservatório (m);

- Nível do Reservatório (m);

- Nível do Canal de Fuga (m);

- Queda Líquida (m);

Resultando em um total de 42258 dados, sendo 7043 de cada variável em um intervalo de tempo horário, retirados de estações hidrométricas, de toda a instrumentação envolvida na Pequena Central Hidrelétrica e do Centro de Operação que controla o empreendimento.

A grande quantidade de informações coletadas permite a modelagem de um sistema nebuloso robusto e confiável.

\subsection{Modelagem do Sistema Nebuloso}

O projeto do sistema nebuloso foi desenvolvido após a definição do ambiente em que as variáveis linguísticas estão inseridas, associadas ao sistema de controle de nível do reservatório, remetendo então a determinação dos níveis de quantização das variáveis de entrada do controlador. Isto permitiu definir o número de termos primários e graus de pertinência dos conjuntos que envolvem cada variável e a seguir, formular as regras que formam o algoritmo do sistema de apoio à decisão desenvolvido. Posteriormente, a definição dos limites que as variáveis estarão atreladas , o método de inferência e a forma de "defuzzificação" das variáveis incluindo a sua atuação no sistema.

A única variável de entrada do sistema medida indiretamente, através de cálculo é a variação do nível do reservatório, conforme segue abaixo:

$\mathrm{DNR}=\mathrm{NR}(\mathrm{t}+1)-\mathrm{NR}(\mathrm{t})$

Após a análise temporal que as variáveis sofreram durante a operação real do período das amostras, pode-se consolidar o conhecimentos necessário para o entendimento do processo de operação dos dados. Utilizando da Matriz de Correlação de Nij variáveis foram observadas as influências que uma variável possui sobre a outra. Através do coeficiente de correlação de Spearman que expressa a intensidade e o sentido da relação entre duas variáveis, podendo este variar entre -1 e +1 . Segundo Rodrigues (2010) não é necessário que as relações entre variáveis sejam lineares para a determinação desse coeficiente, desde que elas sejam ordinais. 
Tabela1 - Matriz de Correlação - Matriz calculada para se obter a relação entre as variáveis utilizadas nas simulações.

\begin{tabular}{|c|c|c|c|c|c|c|c|c|}
\hline & NR & NJ & NL & PL & DNR & Q & QT & PT \\
\hline NR & 1,00 & 0,58 & 0,88 & 0,01 & $-0,09$ & 0,64 & 0,67 & 0,67 \\
\hline NJ & 0,58 & 1,00 & 0,24 & 0,00 & $-0,22$ & 0,64 & 0,73 & 0,73 \\
\hline NL & 0,88 & 0,24 & 1,00 & 0,00 & 0,00 & 0,46 & 0,45 & 0,45 \\
\hline PL & 0,01 & 0,00 & 0,00 & 1,00 & 0,11 & 0,08 & 0,06 & 0,06 \\
\hline DNR & $-0,09$ & $-0,22$ & 0,00 & 0,11 & 1,00 & 0,08 & $-0,27$ & $-0,27$ \\
\hline Q & 0,64 & 0,64 & 0,46 & 0,08 & 0,08 & 1,00 & 0,81 & 0,81 \\
\hline QT & 0,67 & 0,73 & 0,45 & 0,06 & $-0,27$ & 0,81 & 1,00 & 1,00 \\
\hline PT & 0,67 & 0,73 & 0,45 & 0,06 & $-0,27$ & 0,81 & 1,00 & 1,00 \\
\hline
\end{tabular}

NR (Nível do Reservatório), NJ (Nível do Canal de Fuga), NL (Queda Líquida), PL (Pluviometria), DNR (Variação no Nível do Reservatório), Q(Vazão Afluente), QT (Vazão Turbinada), PT (Potência Total).

Abaixo estão os critérios para análise dos resultados obtidos com a Matriz de Correlação, conforme Rodrigues 2010 .

- Se Nij < 0,2, a correlação é desconsiderada;

- Se 0,20< Nij < 0,40, a correlação é fraca;

- Se 0,40 < Nij < 0,60, a correlação é moderada;

- Se $0,60<\mathrm{Nij}<0,80$, a correlação é forte;

- Se Nij > 0,80, a correlação é muito forte.

Conclui-se da Matriz de Correlação que algumas variáveis não possuem correlação direta que seja relevante, como a Pluviometria, porém existem relações com alto grau de correlação como é o caso Vazão Afluente e o Nível do Reservatório.

Questionários e entrevistas com especialistas da área foram realizadas, com o objetivo de entender os procedimentos para a operação da $\mathrm{PCH}$, aprimorando o conhecimento da pesquisa na modelagem do problema. As variáveis tomadas como base para definição dos patamares (valores limites de máximos e mínimos que as variáveis podem alcançar) das funções de pertinência foram obtidos através do modelo reduzido do fabricante da turbina da $\mathrm{PCH}$ em questão, que é representado através da Curva de Colina da Turbina, e dos os estudos ambientais da região, que seguem a Resolução 01, CONAMA (1986), Resolução 06, CONAMA (1987), Resolução 279, CONAMA (2001) e Resolução 302, CONAMA (2002).

O controle do nível de água do reservatório está vinculado à variação na abertura e fechamento do distribuidor da Turbina, sendo esse realizado pelo Regulador de Velocidade. Essa abertura do distribuidor possui uma relação com a Potência Selecionada em cada Unidade Geradora. Conclui-se que a Potência Selecionada, Abertura do Distribuidor e a Vazão Turbinada influenciam na mesma proporção o Nível do reservatório, podendo utilizar-se de apenas uma dessas variáveis.
A Figura 1 mostra as funções de pertinência adotadas para a variável Nível do Reservatório, divididas em NRMB (nível do reservatório muito baixo), NRB (nível do reservatório baixo), NRM (nível do reservatório médio), NRA nível do (reservatório alto), NRMA (nível do reservatório muito alto).

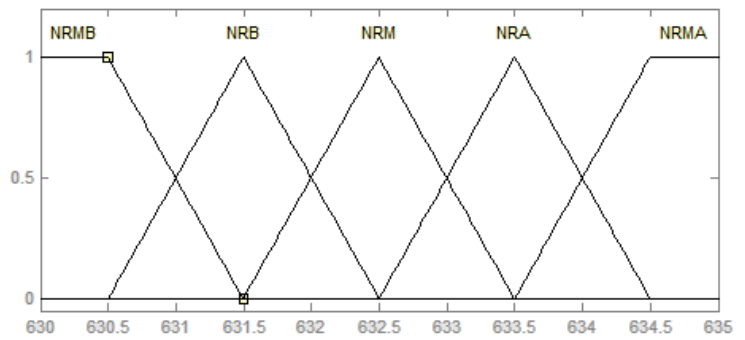

Para a variável Potência Selecionada foram adotadas as funções de pertinência PMB (potência muito baixa), PB (potência baixa), PM (potência média), PA (potência alta), e PMA (potência muito alta), conforme mostra a Figura 2.

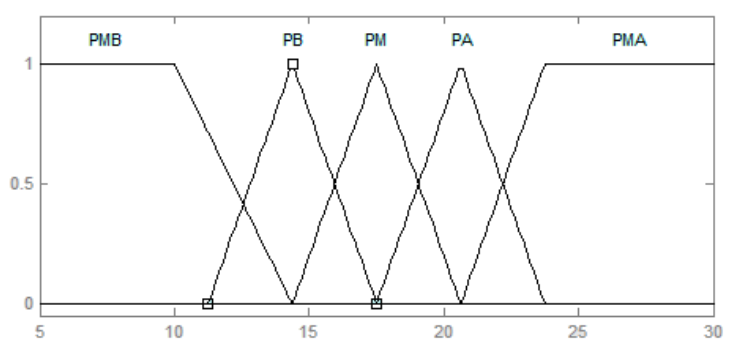

Na Figura 3 tem-se as funções de pertinência para a Vazão Afluente, QMB (vazão muito baixa), QB (vazão baixa), QM (vazão média), QA (vazão alta), e QMA (vazão muito alta).

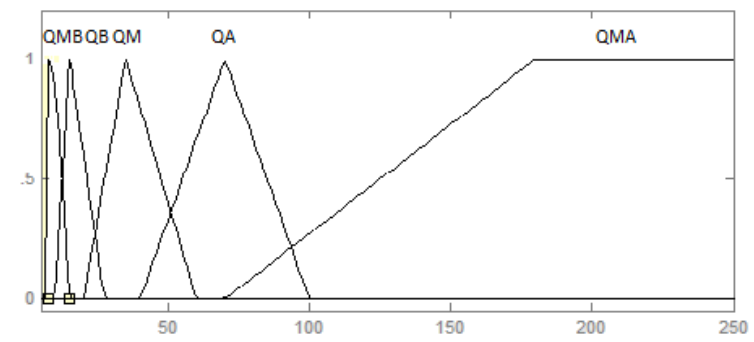

A Queda Líquida é dada por NLMP (queda líquida muito pequena), NLP (queda líquida pequena), NLM (queda líquida media), NLG (queda líquida grande), e NLMG (queda líquida muito grande) (ver Figura 4). 


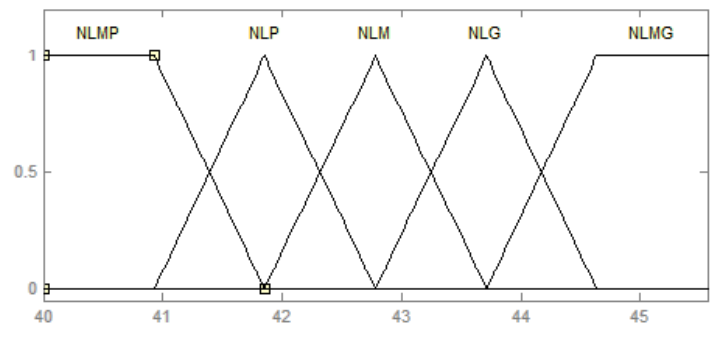

A variação do Nível do Reservatório é dada por DNMB (variação do nível muito baixa), DNB (variação do nível baixa), DNM (variação do nível média), DNA (variação do nível alta), (variação do nível muito alta) (ver Figura 5).

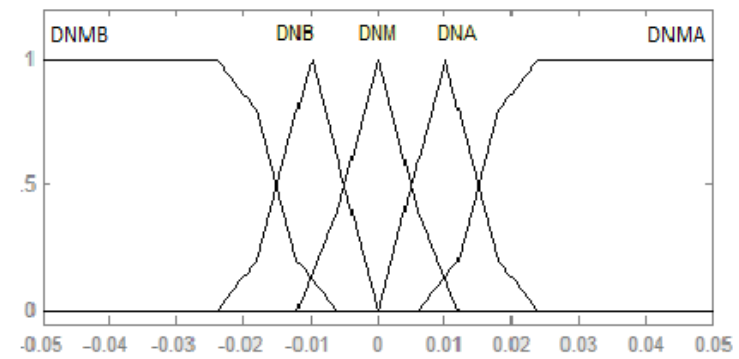

O Nível do Canal de fuga tem como variáveis o NJMB (nível do canal de fuga muito baixo), NJB (nível do canal de fuga baixo), NJM (nível do canal de fuga médio), NJA (nível do canal de fuga alto), e o NJMA (nível do canal de fuga muito alto) (Figura $6)$.

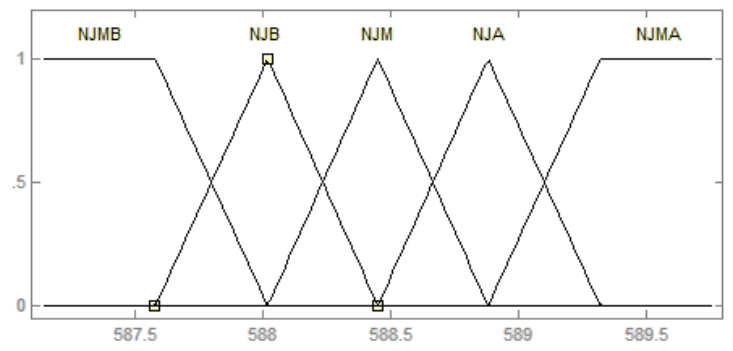

As Tabelas 2-5 indicam todas as regras criadas para dar suporte ao sistema de decisão desenvolvido. Ao todo foram definidas cem regras que suportam a operação da $\mathrm{PCH}$ em questão.

Tabela 2 - Primeiro Conjunto de Regras

\begin{tabular}{|c|c|c|c|c|c|c|}
\hline \multirow{2}{*}{\multicolumn{2}{|c|}{ 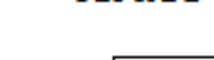 }} & \multirow{2}{*}{\multicolumn{5}{|c|}{ NÍVEL DO RESERVATÓRIO }} \\
\hline & & & & & & \\
\hline & 1 & NRMB & NRB & NRM & NRA & 8 \\
\hline \multirow{5}{*}{ 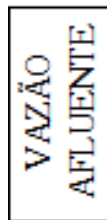 } & & & & & & \\
\hline & & & PI & & & \\
\hline & $\mathrm{QN}$ & $\mathrm{M}$ & $\mathrm{PA}$ & & & PMA \\
\hline & $\mathrm{QA}$ & & $\mathrm{PA}$ & & & \\
\hline & QMA & $\mathrm{PA}$ & PMA & PMA & PMA & PMA \\
\hline
\end{tabular}

Tabela 3 - Segundo Conjunto de Regras

\begin{tabular}{|c|c|c|c|c|c|c|}
\hline & & \multicolumn{5}{|c|}{ QUEDA LÍQUIDA } \\
\hline & 2 & NLMP & NLP & NLM & NLG & NLMG \\
\hline \multirow{5}{*}{ 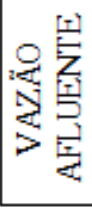 } & QMB & PMB & PB & PM & PA & PMA \\
\hline & $Q B$ & PB & PM & PA & PA & PMA \\
\hline & $\mathrm{QM}$ & PM & PA & PA & PA & PMA \\
\hline & QA & PA & PA & PA & PMA & PMA \\
\hline & QMA & PMA & PMA & PMA & PMA & PMA \\
\hline
\end{tabular}

Tabela 4 - Terceiro Conjunto de Regras

\begin{tabular}{|c|c|c|c|c|c|c|}
\hline & \multirow{2}{*}{\multicolumn{5}{|c|}{ NÍVEL JUSANTE }} \\
\hline & & & & & & \\
\hline & 3 & NJMB & NJB & NJM & NJA & NJMA \\
\hline \multirow{5}{*}{ 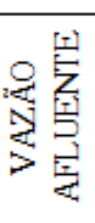 } & QMB & PMB & PB & PM & PM & $\mathrm{PA}$ \\
\hline & $\mathrm{QB}$ & $\mathrm{PB}$ & PB & $\mathrm{PM}$ & $\mathrm{PA}$ & \\
\hline & $\mathrm{QM}$ & PM & PM & $\mathrm{PA}$ & $\mathrm{PA}$ & PMA \\
\hline & $\mathrm{QA}$ & PM & $\mathrm{PA}$ & $\mathrm{PA}$ & PMA & PMA \\
\hline & QMA & $\mathrm{PA}$ & $\mathrm{PA}$ & PMA & PMA & PMA \\
\hline
\end{tabular}

Tabela 5 - Quarto Conjunto de Regras

\begin{tabular}{|c|c|c|c|c|c|c|}
\hline & \multicolumn{5}{|c|}{ NIVEL DO RESERVATÓRIO } \\
\hline & 4 & NRMB & NRB & NRM & NRA & NRMA \\
\hline \multirow{5}{*}{ 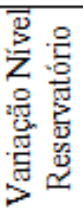 } & DNMB & PMB & PB & PM & PA & PA \\
\hline & $\mathrm{DNB}$ & PB & PM & PM & PA & $\mathrm{PA}$ \\
\hline & DNM & PM & PM & $\mathrm{PA}$ & $\mathrm{PA}$ & PMA \\
\hline & DNA & PM & PM & $\mathrm{PA}$ & PMA & PMA \\
\hline & DNMA & PM & PA & PMA & PMA & PMA \\
\hline
\end{tabular}

\section{Resultados}

A figura 7 compara as Potências Reais Selecionadas pelo operador da PCH e as Potências Selecionadas pelo Sistema Nebuloso, ambas utilizando os mesmos valores das variáveis de entrada. No eixo das abscissas estão os números de potencias selecionadas nos dois sistemas, totalizando 6309 potências selecionadas pelo operador e 6309 potências selecionadas pelo sistema nebuloso, já no eixo das ordenadas estão os valores das potências selecionadas em MW.

Os resultados se mostraram promissores, pois a energia elétrica acumulada gerada através do controle manual realizado pelo operador da Pequena Central Hidrelétrica foi de aproximadamente 121,745MWh. Já com o suporte decisório do sistema nebuloso o total de energia gerada prevista caso as máquinas fossem operadas dentro dos limites sugeridos pelo sistema desenvolvido seria de $127,909 \mathrm{MWh}$, representando um acréscimo de $5 \%$ na geração. 


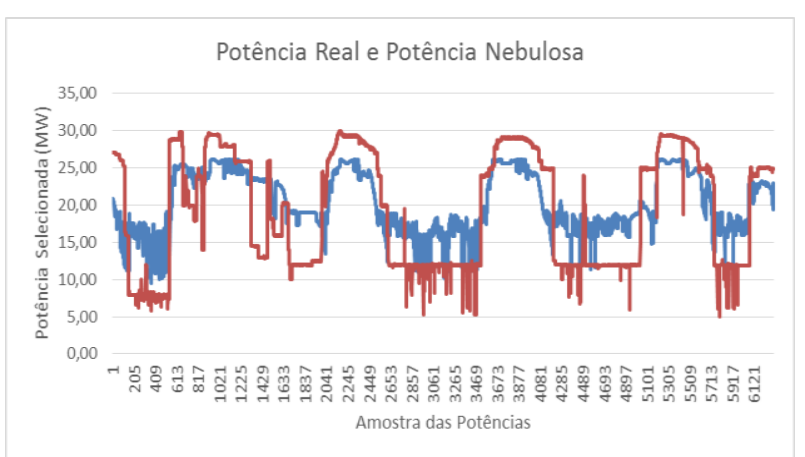

Figura 7 - Potências Selecionadas pelo Operador e Pelo Sistema Nebuloso

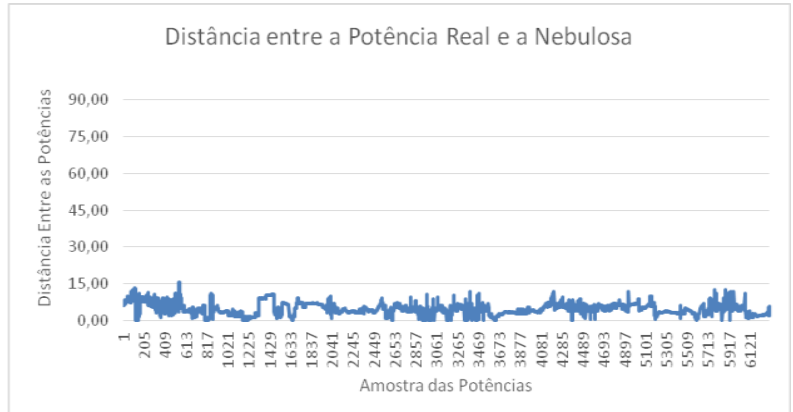

Figura 8 - Distância entre a Potência Real e a Nebulosa

A Figura 8 mostra distância entre as potências selecionadas reais e pelo sistema nebuloso através da fórmula (2).

$\mathrm{R}=((\mathrm{Pr}-\mathrm{Pn}) 2) 1 / 2(2)$

Onde Pr é a Potência Real selecionada pelo operador, Pn é a Potência Selecionada pelo sistema Nebuloso e R a distância entre as potências.

\section{Conclusão}

Os resultados obtidos nesse trabalho apresentaram-se interessantes. Foram utilizados para as simulações apenas os dados em que a Pequena Central Hidrelétrica estava com disponibilidade total para geração de energia elétrica, desconsiderando-se eventuais paradas. A potência selecionada não sofreu interferência alguma de possíveis perturbações externas do sistema elétrico, pois tais variações poderiam mascarar os resultados. Apesar disso, atingiu-se um percentual considerável de ganho na produção de energia através da operação simulada da usina.

Foi possível também verificar a interdependência entre as diversas variáveis de entrada do sistema nebuloso, além de propiciar uma efetiva transferência de conhecimento entre os controladores reais e o sistema nebuloso desenvolvido, através do grande conjunto de regras proposto.

\section{Referências}

Coen, M. (1974). Macchine Idrauliche. SIGNORELLI/MILANO.
Conama. (1986). Resolução Conama $\mathrm{n}^{\circ} 1$, Critérios básicos e diretrizes gerais para avaliação do impacto ambiental, pp.2548-2549. Disponível em: $<>$.

http://www.iap.pr.gov.br/modules/conteudo/conte udo.php?conteudo $=985$. Curitiba, Paraná, Brasil. Acessado em: 30 abril de 2012.

Niimura, T.; Yasuda K.; Yokoyama R. (1995). Water Level Control of Small-scale Hydro-Generating Units by Fuzzy Logic. In IEEJ National Convention Record, No. 2483.

Russel, S. O.; Campbell, P. F. (1996). Reservoir Operating Rules with Fuzzy Programming. Journal of Water Resources Planning and Management 1996.122, pp165-170. DOI: 10.1061/(ASCE)0733-9496(1996)122:3(165)

Rodrigues, W.C. (2010). Estatística Aplicada .Rio de Janeiro - RJ, 49p.

Takagi, T.; Sugeno, M. (1985). Fuzzy Identification of System and its Applicaiton to Modeling and Control. In IEEE Transactions on Systems, Man and Cybernetics. Fev. 1985, pp 116-132. DOI: 10.1109/TSMC.1985.6313399

Zadeh, L. A. (1973). Outline of a New Approach to the Analysis of Complex Systems and Decision Processes. In IEEE Transactions on Systems, Man and Cybernetics, Berkeley, Jan. 1973, pp 28 - 44 DOI: 10.1109/TSMC.1973.5408575 